\title{
Memory effects in quantum channel discrimination
}

\author{
Giulio Chiribella, Giacomo M. D'Ariano, and Paolo Perinotti \\ QUIT - Quantum Information Theory Group, Dipartimento di Fisica \\ "A. Volta" Università di Pavia, via A. Bassi 6, I-27100 Pavia, Italy.
}

(Dated: May 28, 2018)

\begin{abstract}
We consider quantum-memory assisted protocols for discriminating quantum channels. We show that for optimal discrimination of memory channels, memory assisted protocols are needed. This leads to a new notion of distance for channels with memory. For optimal discrimination and estimation of sets of independent unitary channels memory-assisted protocols are not required.
\end{abstract}

PACS numbers:

The problem of discrimination between quantum channels has been recently considered in quantum information

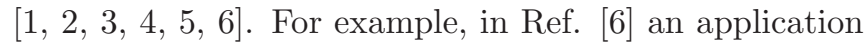
of discrimination of unitary channels as oracles in quantum algorithms is suggested. The optimal discrimination is achieved by applying the unknown channel locally on some bipartite input state of the system with an ancilla, and then performing some measurement at the output. A natural extension to multiple uses is obtained by applying the uses in parallel to a global input state. However, more generally, one can apply the uses partly in parallel and partly in series, even intercalated with other fixed transformations, as in Ref. [7]. Indeed, due to its intrinsic causally ordered structure, the memory channel can be used either in parallel or in a causal fashion (see Fig. 11). In this Letter we show that this causal scheme is necessary when the multiple uses are correlated-i. e. for memory channels - whereas it is not needed for independent uses of unitary channels (the case of non unitary channels remains an open problem). Memory channels

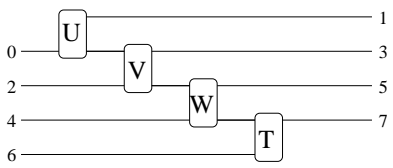

a)

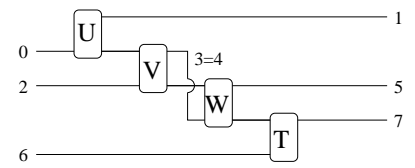

b)
FIG. 1: Different usage schemes of a general memory channel, where the boxes $U, V, W, T$ denote interactions of systems with ancillae. a) Parallel scheme (a multipartite input state is evolved through the channel). b) A particular case of causal scheme, (the output of some use of the channel is fed into a successive use).

[8, 9, 10, 11, 12] attracted increasing attention in the last years. They are quantum channels whose action on the input state at the $n$-th use can depend on the previous $n-1$ uses through a quantum ancilla. The problem of optimal discriminability of two memory channels is relevant for assessing that a cryptographic protocol is concealing [13] and for minimization of oracle calls in quantum algorithms.

We will provide an example showing that a pair of memory channels can be perfectly discriminabile, even though they never provide orthogonal output states when applied to the same global input state. This new causal setup provides the most general discrimination scheme for multiple quantum channels, and this fact leads to a new notion of distance between channels.

In the case of two unitary channels, optimal parallel discrimination with $N$ uses was derived in Ref. 11, 2, and in Ref. [5] a causal scheme without entanglement was proved to be equivalently optimal. In the following, we will prove the optimality of both schemes for discrimination of unitaries. We will generalize this result to discrimination of sequences of unitaries, and to estimation with multiple copies. Differently from the case of memory channels, we will prove that for all these examples causal schemes are not necessary.

It is convenient to represent a channel $\mathscr{C}$ by means of its Choi operator $C$ defined as follows

$$
C:=\mathscr{C} \otimes \mathscr{I}(|I\rangle\rangle\langle\langle I|),
$$

for a channel $\mathscr{C}$ with input/output states in $\mathcal{H}_{\text {in/out }}$, respectively, where $|I\rangle\rangle:=\sum_{n}|n\rangle|n\rangle \in \mathcal{H}_{\text {in }}^{\otimes 2},\{|n\rangle\}$ being an orthonormal basis for $\mathcal{H}_{\text {in }}$. In this representation complete positivity of $\mathscr{C}$ is simply $C \geq 0$ ) and the tracepreserving constraint is $\operatorname{Tr}_{\text {out }}[C]=I_{\text {in }}$.

In a memory channel with $N$ inputs and $N$ outputs labeled as in Fig. 11, the causal independence of output $2 n+1$ on input $2 m$ with $m>n$ is translated to the following recursive property [7] of the Choi operator $C=$ : $C^{(N)}$

$$
\operatorname{Tr}_{2 n-1}\left[C^{(n)}\right]=I_{2 n-2} \otimes C^{(n-1)}, \quad \forall 1 \leq n \leq N,
$$

where conventionally $C^{(0)}=1$. A tester is a set of positive operators $P_{i} \geq 0$ such that the probability of outcome $i$ while testing the channel $\mathscr{C}$ is provided by the generalized Born rule

$$
p(i \mid \mathscr{C}):=\operatorname{Tr}\left[P_{i} C\right] .
$$

The notion of tester is an extension of that of POVM, which describes the statistics of customary measurements on quantum states. The normalization of probabilities for testers on memory channels with $N$ input-output systems 
is equivalent to the following recursive property, analogous to that in Eq. (2)

$$
\begin{aligned}
& \sum_{i} P_{i}=I_{2 N-1} \otimes \Xi^{(N)}, \\
& \operatorname{Tr}_{2 n-2}\left[\Xi^{(n)}\right]=I_{2 n-3} \otimes \Xi^{(n-1)}, \quad \forall 2 \leq n \leq N, \\
& \operatorname{Tr}\left[\Xi^{(1)}\right]=1 .
\end{aligned}
$$

One can prove [7] that any tester can be realized by a concrete measurement scheme of the class represented in Fig. 2. Mathematical structures analogous to Eqs. (2)

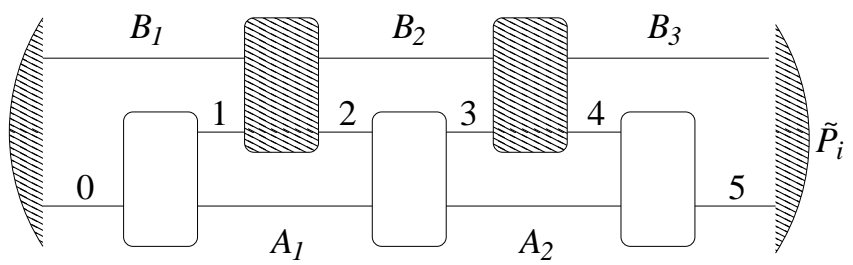

FIG. 2: The most general scheme for the connection of a memory channel to a quantum circuit corresponding to a tester. The memory channel is represented by its isometric gates (white boxes) which denote interaction of quantum systems (inputs are labeled by even integers and outputs by odd integers) with the ancillae $A_{1}$ and $A_{2}$. The tester is represented by dashed boxes, including the preparation phase (joint input state of system 0 and ancilla $B_{1}$ ) and the final measurement stage represented by the POVM $\left\{\tilde{P}_{i}\right\}$.

and (4) have been introduced in Ref. [14 to describe strategies in a quantum game.

Every tester $\left\{P_{i}\right\}$ can be written in terms of a usual $\operatorname{POVM}\left\{\tilde{P}_{i}\right\}$ as follows

$$
P_{i}=\left(I \otimes \Xi^{(N) \frac{1}{2}}\right) \tilde{P}_{i}\left(I \otimes \Xi^{(N) \frac{1}{2}}\right),
$$

and for every memory channel $\mathscr{C}$ the generalized Born rule rewrites as the usual one in terms of the state

$$
\tilde{C}:=\left(I \otimes \Xi^{(N) \frac{1}{2}}\right) C\left(I \otimes \Xi^{(N) \frac{1}{2}}\right) .
$$

The state $\tilde{C}$ corresponds to the output system-ancilla state in Fig 2 after the evolution through all boxes of both the tester and the memory channel, on which the final POVM $\left\{\tilde{P}_{i}\right\}$ is performed 15 .

The standard discriminability criterion for channels is the following. Two channels $\mathscr{C}_{0}$ and $\mathscr{C}_{1}$ on a $d$ dimensional system are perfectly discriminable if there exists a pure state $|\Psi\rangle\rangle$ in dimension $d^{2}$ such that $\mathscr{C}_{i} \otimes$ $\mathscr{I}(|\Psi\rangle\rangle\langle\langle\Psi|$ ) with $i=0,1$ are orthogonal (every joint mixed state with an ancilla of any dimension can be purified with an ancilla of dimension $d$ ). Here we use the notation $|\Psi\rangle\rangle:=\sum_{m, n}|m\rangle|n\rangle$ which associates an operator $\Psi$ to a bipartite vector. It is easy to see that the orthogonality between the two output states is equivalent to the following condition [16]

$$
C_{0}(I \otimes \rho) C_{1}=0,
$$

where $\rho:=\Psi^{*} \Psi^{T}$, where $\Psi^{*}$ and $\Psi^{T}$ denote the complex conjugate and transpose of $\Psi$ in the canonical basis $\{|n\rangle\}$, respectively. The criterion in Eq. (7) is too restrictive for memory channels. Indeed, the correct condition for perfect discriminability of two memory channels $\mathscr{C}_{i}$ with $i=0,1$ is equivalent to the existence of a tester $\left\{P_{i}\right\}$ with $i=0,1$, such that

$$
\operatorname{Tr}\left[P_{i} C_{j}\right]=\delta_{i j},
$$

which means that the two channels can be perfectly discriminated by a measurement scheme as that of Fig. 2. Using Eqs. (5) and (6), Eq. (8) becomes $\operatorname{Tr}\left[\tilde{P}_{i} \tilde{C}_{j}\right]=\delta_{i j}$, whence the states $\tilde{C}_{i}$ with $i=0,1$ are orthogonal, and the same derivation as for Eq. (7) leads to

$$
C_{0}\left(I \otimes \Xi^{(N)}\right) C_{1}=0,
$$

with $\Xi^{(N)}$ as in Eq. (凷). In Eq. (9) the identity operator acts only on space $2 N-1$, differently from Eq. (7) where it acts on all output spaces.

It is interesting to analyze the special case of memory channels made of sequences of independent channels $\left\{\mathscr{C}_{i j}\right\}_{1 \leq j \leq N}$ and $i=0,1$ (in Fig. 2, the memory channel is replaced by an array of channels without the ancillas $A_{1}$ and $A_{2}$ ). The condition for perfect discriminability is the same as Eq. (9) with $C_{0}$ and $C_{1}$ replaced by $\bigotimes_{j} C_{i j}$ for $i=0,1$, respectively. In terms of a Kraus form $\mathscr{C}_{i}=\sum_{j} K_{i j} \cdot K_{i j}^{\dagger}$ Eq. (9) becomes the orthogonality condition $\left\langle\left\langle K_{0 j}\left|\left(I \otimes \Xi^{(N)}\right)\right| K_{1 k}\right\rangle\right\rangle=0$, which for the sequences of maps becomes

$$
\bigotimes_{l=1}^{N}\left\langle\left\langle K_{0 j_{l}}^{l}\left|\left(I \otimes \Xi^{(N)}\right) \bigotimes_{m=1}^{N}\right| K_{1 k_{m}}^{m}\right\rangle\right\rangle=0 .
$$

for all choices of indices $(j),(k)$, where $K_{i j}^{m}$ are the Kraus operators for the channel $\mathscr{C}_{i m}$. For sets composed by single channels $\mathscr{C}_{i}$ with $i=0,1$, the condition becomes simply the existence of a state $\rho$ such that

$$
\operatorname{Tr}\left[\rho K_{0 j}^{\dagger} K_{1 k}\right]=0, \quad \forall j, k,
$$

and the minimum rank of such state $\rho$ determines the amount of entanglement required for discrimination.

We now provide an example of memory channels that cannot be discriminated by a parallel scheme, but can be discriminated with a tester. Each memory channel has two uses, and is denoted as $\mathscr{C}_{i}=\mathscr{W}_{i} \circ \mathscr{Z}_{i}$ for $i=0,1$, where the two uses $\mathscr{W}_{i}$ and $\mathscr{Z}_{i}$ are connected only through the ancilla $A$, and $\mathscr{W}_{i}$ has input 0 and output $A$ and 1 , and $\mathscr{Z}_{i}$ has input $A$ and 2 and output 3 . The first use $\mathscr{W}_{0}$ of $\mathscr{C}_{0}$ is the channel with $d$-dimensional input and fixed output

$$
\mathscr{W}_{0}(\rho)=\frac{1}{d^{2}} \sum_{p, q=0}^{d-1}|p, q\rangle\langle p, q|\otimes| p, q\rangle\langle p, q|,
$$


$|p, q\rangle$ being an orthonormal basis in a $d^{2}$ dimensional Hilbert space. The second use $\mathscr{Z}_{0}$ of $\mathscr{C}_{0}$ is given by

$$
\mathscr{Z}_{0}(\rho)=\sum_{p, q=1}^{d-1} W_{p, q} \operatorname{Tr}_{A}\left[\rho\left(I_{2} \otimes|p, q\rangle\langle p, q|\right)\right] W_{p, q}^{\dagger},
$$

where the unitaries $W_{p, q}:=Z^{p} U^{q}$ are the customary shift-and-multiply operators, with $Z|n\rangle=|n+1\rangle$ and $U|n\rangle=e^{\frac{2 \pi i}{d} n}|n\rangle$. The second channel $\mathscr{C}_{1}$ is given by

$$
\mathscr{W}_{1}(\rho)=\frac{I}{d^{2}}, \quad \mathscr{Z}_{1}(\rho)=|0\rangle\langle 0| .
$$

We will now show that the two channels are discriminable with a casual setup and not with a parallel one. Their Choi operators are

$$
\begin{aligned}
& \left.C_{0}=\frac{1}{d^{2}} \sum_{p, q=1}^{d-1}|p, q\rangle\left\langle p,\left.q\right|_{1} \otimes \mid W_{p, q}\right\rangle\right\rangle\left\langle\left\langle\left. W_{p, q}\right|_{32} \otimes I_{0},\right.\right. \\
& C_{1}=\frac{1}{d^{2}} I_{1}^{\otimes 2} \otimes|0\rangle\left\langle\left. 0\right|_{3} \otimes I_{02},\right.
\end{aligned}
$$

where the output spaces 1,3 have dimension $d^{2}$ and $d$, respectively. Suppose that the channels are perfectly discriminable, then by Eq. (7) there exists $\rho$ such that

$$
C_{0}\left(I_{13} \otimes \rho_{02}\right) C_{1}=C_{0} C_{1}\left(I_{13} \otimes \rho_{02}\right)=0,
$$

where the second equality comes from the expression of $C_{1}$ in Eq. (15). Tracing both sides on the output spaces 1 and 3 one has $\operatorname{Tr}_{13}\left[C_{0} C_{1}\right] \rho=0$. However,

$$
\operatorname{Tr}_{13}\left[C_{0} C_{1}\right]=\frac{I}{d^{2}}
$$

whence $\rho=0$. This proves by contradiction that the criterion in Eq. (7) - corresponding to parallel discrimination schemes - is not satisfied by channels $\mathscr{C}_{0}$ and $\mathscr{C}_{1}$. We will now show a simple causal scheme which allows perfect discrimination of the same channels. The first use of the channel is applied to any state $|\psi\rangle\langle\psi|$, then the measurement with $\operatorname{POVM}\{|p, q\rangle\langle p, q|\}$ is performed at the output on system 1. Depending on the outcome $\bar{p}, \bar{q}$, the second use of the channel is applied to the state $W_{\bar{p}, \bar{q}}^{\dagger}|1\rangle\langle 1| W_{\bar{p}, \bar{q}}$. It is clear that the output of channel $\mathscr{Z}_{0}$ is the state $|1\rangle\langle 1|$, whereas the output of $\mathscr{Z}_{1}$ is $|0\rangle\langle 0|$.

This example highlights the need of using a causal scheme in order to discriminate between memory channels. The causal discriminability criterion (9) implies a notion of distance between memory channels different from the usual distance between channels. Indeed, the discriminability criterion (7) between channels corresponds to the cb-norm distance [17, 18, 19]. The latter can be rewritten as follows (see e.g. Ref [3])

$$
\begin{aligned}
& D_{c b}\left(\mathscr{C}_{0}, \mathscr{C}_{1}\right)=\max _{\rho}\left\|\left(I \otimes \rho^{\frac{1}{2}}\right) \Delta\left(I \otimes \rho^{\frac{1}{2}}\right)\right\|_{1}, \\
& \Delta:=C_{0}-C_{1},
\end{aligned}
$$

where the maximum is over all states $\rho$, and $\|X\|_{1}:=\operatorname{Tr}\left[\sqrt{X^{\dagger} X}\right]$ denotes the trace-norm. One has $D_{c b}\left(\mathscr{C}_{0}, \mathscr{C}_{1}\right) \leq 2$, with the equal sign for perfectly discriminable channels. For memory channels the discriminability criterion (7) corresponds to the new distance

$$
D\left(\mathscr{C}_{0}, \mathscr{C}_{1}\right):=\max _{\Xi^{(N)}}\left\|\left(I \otimes \Xi^{(N) \frac{1}{2}}\right) \Delta\left(I \otimes \Xi^{(N) \frac{1}{2}}\right)\right\|_{1},
$$

where the maximum is over all $\Xi^{(N)}$ satisfying conditions (顿. For $N=1$ this notion reduces to the usual distance in Eq. (18).

The easiest application of testers is the discrimination of sequences of unitary channels $\left(T_{j}\right)$ and $\left(V_{j}\right)$, with $j=$ $1, \ldots, N$. Without loss of generality we can always reduce to the discrimination of the sequence $\left(U_{j}\right):=\left(T_{j}^{\dagger} V_{j}\right)$ from the constant sequence $(I)$. Let us first consider the case of sequences of two unitaries. By referring to the scheme in Fig. 2 we can restate the problem as the discrimination of $W^{\dagger}\left(U_{1} \otimes I\right) W\left(U_{2} \otimes I\right)$ from $I$ on a bipartite system, where $W$ describes the interaction with an ancillary system. It is well known that optimal discriminability of a unitary $X$ from the identity is related to the angular spread $\Theta(X)$, defined as the maximum relative phase between two eigenvalues of $X$ [1, 2]. Apart from the degenerate case in which $X$ has only two different eigenvalues, the discriminability of $X$ from $I$ is given by the quantity $\max \{0, \cos \Theta(X) / 2\} \geq 0$, which is zero for $\Theta(X) \geq \pi$, corresponding to perfect discriminability. Since unitary conjugation preseves $\Theta(X)$ and the angular spread of the product of two unitaries $X, Y$ satisfies the following bound [20]

$$
\Theta(X Y) \leq \Theta(X)+\Theta(Y)
$$

and finally $\Theta(X \otimes Y)=\Theta(X)+\Theta(Y)$, one has that $\Theta\left[W^{\dagger}\left(U_{1} \otimes I\right) W\left(U_{2} \otimes I\right)\right] \leq \Theta\left(U_{1} \otimes U_{2}\right)$, then no causal scheme can outperform the parallel one. By induction, one can prove that this is true for sequences of any length $N$. Indeed, defining $X_{N-1}$ as the product of the tester unitaries alternated with $U_{j} \otimes I$ for $1 \leq j<N$, if $\Theta\left(X_{N-1}\right)=\Theta\left(\otimes_{j=1}^{N-1} U_{j}\right)$ holds true, then it holds also for $N$, due to Eq. (20). By the same argument, one can also prove that the sequential scheme of Ref. [3] equals the performances of the parallel scheme, since there always exists $T$ such that $\Theta\left(U T V T^{\dagger}\right)=\Theta(U \otimes V)$ (indeed it is sufficient that $T$ transforms the eigenbasis of $V$ into that of $U$, suitably matching the eigenvalues). Therefore, the schemes of Refs. 11, 2, 5] are optimal also for discriminating sequences of unitaries. Notice that this also includes the case of discrimination of two different permutations of a sequence of unitary transformations.

Another situation in which a parallel scheme already performs optimally is the case of estimation of unitary transformations $U_{g}, g \in G$ which make a unitary representation of the group $G$. For $N$ uses of the unitary $U_{g}$ 
the Choi operator in this case is

$$
\left.R_{g}^{(N)}=R_{g}^{\otimes N}, R_{g}=\left(U_{g} \otimes I\right)|I\rangle\right\rangle\left\langle\langle I|\left(U_{g}^{\dagger} \otimes I\right) .\right.
$$

The probability density of estimating $h$ for actual element $g$ is

$$
p(h \mid g)=\operatorname{Tr}\left[P_{h} R_{g}^{(N)}\right]
$$

As a figure of merit for estimation one typically considers a cost function $c(h, g)$ averaged on $h$, with $c(h, g)=$ $c(f h, f g) \forall f \in G$ (the cost depends only on distance, not on specific location)

$$
C_{g}(p)=\int_{G} \mu(\mathrm{d} h) c(h, g) p(h \mid g),
$$

where $\mu(\mathrm{d} g)$ is the invariant Haar measure on $G$. The optimal density $p$ is the one minimizing $\hat{C}(p):=$ $\max _{g \in G} C_{g}(p)$. For every density $p(h \mid g)$ there exists a covariant one $p_{c}(h \mid g)=p_{c}(f h \mid f g) \forall f \in G$ which can be obtained as the average $p_{c}(h \mid g):=\overline{p(f h \mid f g)}$ over $f \in G$ (practically this corresponds to randomly transforming the input before measuring and processing the output accordingly). Since $\hat{C}\left(p_{c}\right)=\bar{C}(p) \leq \hat{C}(p)$, then the optimal density minimizing both costs $\hat{C}$ and $\bar{C}$ can be chosen as covariant. Now, since $p_{c}(h \mid g)=p_{c}\left(e \mid g h^{-1}\right)$ (e denoting the identity element in $G$ ), this means that the optimal tester must be of the covariant form

$$
P_{h}=\left(U_{h} \otimes I\right)^{\otimes N} P_{e}\left(U_{h}^{\dagger} \otimes I\right)^{\otimes N} .
$$

For such $P_{h}$, the normalization $\int_{G} \mu(\mathrm{d} h) P_{h}=I \otimes \Xi^{(N)}$ implies the commutation $\left[I \otimes \Xi^{(N)},\left(U_{h} \otimes I\right)^{\otimes N}\right]=0$, whence the POVM $\tilde{P}_{h}$ in Eq. (5) is itself covariant. The optimal tester problem is then equivalent to the optimal state estimation in the orbit $\left(I \otimes \Xi^{(N) \frac{1}{2}}\right) R_{g}^{(N)}\left(I \otimes \Xi^{(N) \frac{1}{2}}\right)$. This proves that the optimal estimation of $U_{g}$ with $g \in G$ compact group can be reduced to a covariant state estimation problem, and the parallel scheme of Ref. 22] is optimal. The possibility of achieving the same optimal estimation using a sequential scheme as in Ref. 55] remains an open problem, as, more generally, the possibility of minimizing the amount of entanglement used by the tester.

In conclusion, we considered the role of memory effects in the discrimination of memory channels and of customary channels with multiple uses. We used the new notion of tester [7], which describes any possible scheme with parallel, sequential, and combined setup of the tested channels. We provided an example of discrimination of memory channels which cannot be optimized by a parallel scheme, and for which the optimal discrimination is achieved by a sequential scheme. The new testing of memory channels corresponds to a new notion of distance between channels. Finally, we showed that for the purpose of unitary channel discrimination and estimation with multiple uses, memory effects are not needed.

This work has been supported by the EC through the project SECOQC.

[1] G. M. D'Ariano, and P. Lo Presti, M. G. A. Paris, Phys. Rev. Lett. 87, 270404 (2001).

[2] A. Acín, Phys. Rev. Lett. 87, 177901 (2001).

[3] M. F. Sacchi, Phys. Rev. A 71, 062340 (2005).

[4] G. M. D'Ariano, M. F. Sacchi, and J. Kahn, Phys. Rev. A 72, 052302 (2005).

[5] R. Duan, Y. Feng, and M. Ying, Phys. Rev. Lett., 98, 100503, (2007).

[6] A. Chefles, A. Kitagawa, M. Takeoka, M. Sasaki, and J. Twamley, To appear in J. Phys. A: Math. Theor.

[7] G. Chiribella, G. M. D'Ariano, and P. Perinotti, arXiv:0712.1325

[8] C. Macchiavello and G. M. Palma, Phys. Rev. A 65, 050301(R) (2002).

[9] G. Bowen and S. Mancini, Phys. Rev. A 69, 012306 (2004).

[10] D. Kretschmann and R. F. Werner, Phys. Rev. A 72, 062323 (2005).

[11] N. Datta and T. C. Dorlas, J. Phys. A: Math. Theor. 40, 8147-8164 (2007).

[12] M. B. Plenio and S. Virmani, Phys. Rev. Lett. 99, 120504 (2007).

[13] G. M. D'Ariano, D. Kretschmann,D. Schlingemann, and R. F. Werner, Phys. Rev. A 76, 032328 (2007).

[14] G. Gutoski and J. Watrous, in Proceedings of the Thirtynineth Annual ACM Symposium on Theory of Computation (STOC), pag. 565-574, (2007).

[15] This is the delayed measurement principle, stating that for any quantum setup involving measurements, there is an equivalent one in which all measurements are postponed at the final stage

[16] The orthogonality condition is $0=\left(I \otimes \Psi^{T}\right) C_{0}(I \otimes$ $\left.\Psi^{*} \Psi^{T}\right) C_{1}\left(I \otimes \Psi^{*}\right)=H_{0}^{\dagger} H_{0} H_{1}^{\dagger} H_{1}$, with $H_{i}=C_{i}^{\frac{1}{2}}\left(I \otimes \Psi^{*}\right)$, which holds iff $H_{0} H_{1}^{\dagger}=0$.

[17] V. I. Paulsen, Completely Bounded Maps and Operator Algebras, Pitman Research Notes in Math. 146. (Longman Scientific \& Technical, Harlow, 1996).

[18] D. Aharonov, A. Kitaev, and N. Nsan, in Proceedings of the Thirtieth Annual ACM Symposium on Theory of Computation (STOC), pag. 20-30, (1997).

[19] This distance is referred to in the literature as cb-norm distance, since it is induced by the norm of complete boundedness 17 (cb-norm for short, also defined diamond norm in Ref. [18]).

[20] A. M. Childs, J. Preskill, and J. Renes, J. Mod. Opt. 47, 155-176 (2000).

[21] In the degenerate case, if $\Theta(U)+\Theta(V)>\pi$, it is always possible to find $T$ such that $\Theta\left(U T V T^{\dagger}\right)=\pi$.

[22] G. Chiribella, G. M. D'Ariano and M. F. Sacchi, Phys. Rev. A 72, 042338 (2005). 